\title{
Computer simulations and physical modelling of erosion
}

\author{
Christopher S. Stuetzle \& Zhongxian Chen \& Barbara Cutler \\ Rensselaer Polytechnic Institute, Computer Science Dept. \\ W. Randolph Franklin \\ Rensselaer Polytechnic Institute, Electrical, Computer, and Systems Engineering Dept. \\ Jared Gross \& Katrina Perez \& Thomas Zimmie \\ Rensselaer Polytechnic Institute, Civil and Environmental Engineering Dept.
}

\begin{abstract}
Research is being done to study the details and progress of soil erosion on levees and dams, including the formation and progression of rills and gullies on the slopes, and eventually to final breaching. These detailed observations of erosion differ from the typical predictions of only the maximum erosion or scour depths, for example around submerged bridge piers. Computer simulations and geotechnical centrifuge modelling will, in the future, be validated using these observations. For testing, single layer sand models were utilized, and will be followed by clayey and mixed soils, and increased number of layers. The computer simulations will incorporate 3-D Navier-Stokes fluid simulations, and a novel segmented height field extended to allow soil undercuts was developed. The primary intent of the research is to study small-scale erosion on earthen embankments and, ultimately, develop novel and robust erosion software, validated by physical modelling.
\end{abstract}
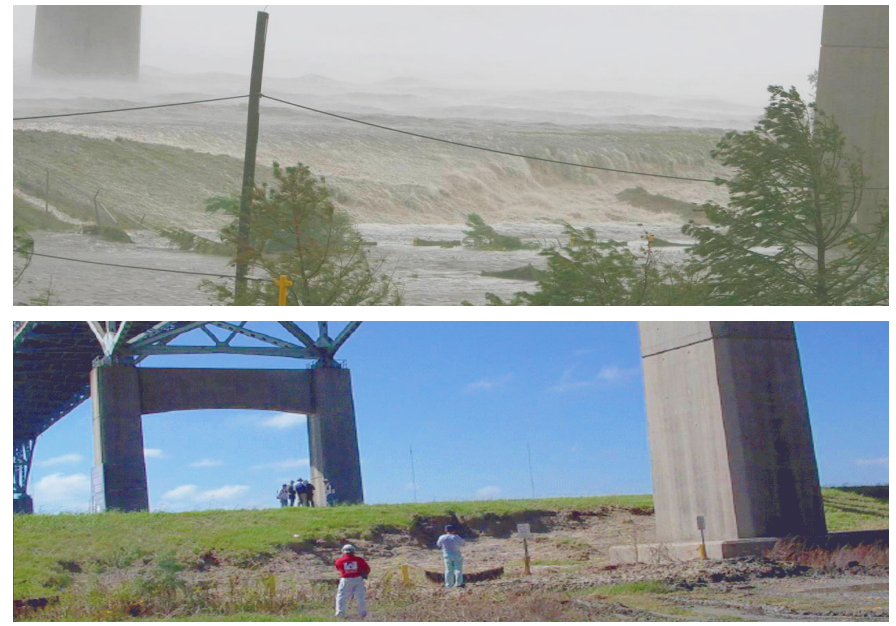

Figure 1: A levee that was overtopped for several hours during the Katrina hurricane but did not fail. The dramatic gouging/scooping on the lower portions of the levee is due to increased water flow at the base of the levee and the nonhomogeneous nature of the embankment.

\section{INTRODUCTION}

The levee failures in the New Orleans area due to Hurricane Katrina received much media attention (Seed et al. 2005). Emphasis in this study is on earthen levees, dams, and embankments. A major cause of failures of such structures is overtopping, which causes erosion to the point of breaching the crest.

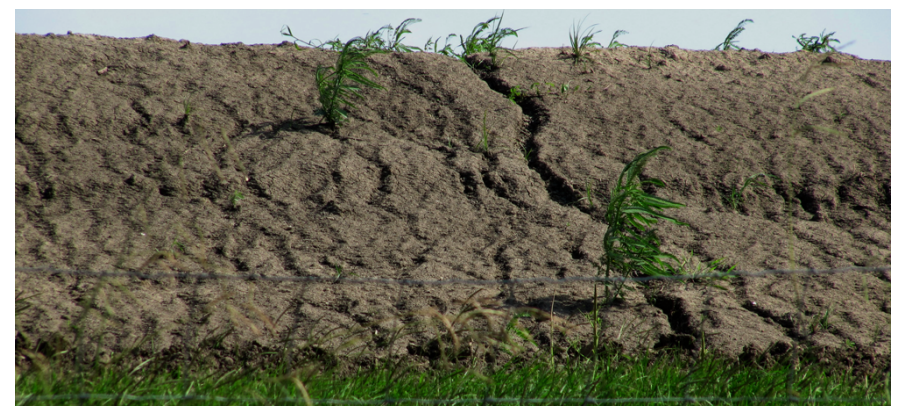

Figure 2: Rills form in an earthen embankment.

Historically, there are many examples of such failures, Katrina levees already noted (Fig. 1). Numerous other examples can be cited, but it is clear that such failures can cause serious loss of lives and economic disaster. Better understanding the ways in which earthen structures that are designed to help protect against flooding fail is an important step toward preventing this largely unnecessary loss of money and lives.

Erosion in this paper refers to hydraulic erosion. We wish to study and eventually be able to simulate small-scale erosion on earthen embankments, with respect to the formation of rills and gullies (Fig. 2). Validation of our simulation will be a primary focus of our research. As a result, we will model levees on a small scale and then perform erosion experiments in a centrifuge. 
Results of our initial experiments are presented later in this paper. To date, our experiments have been done at $1-\mathrm{g}$, using sand. These preparatory experiments are being used to determine the applicable scaling laws that apply to centrifuge erosion models. Eventually, different type soils will be tested and complex geometries and boundary conditions utilized to quantitatively assess the effects of the differing conditions.

\section{REVIEW OF LITERATURE}

Related works in erosion literature can be divided into two groups. Many mathematical models for erosion have been presented in the field of Civil Engineering, for the purpose of studying and predicting the erosion of soil during design and construction of earthen embankments, dams, and levees. Conversely, in the field of Computer Graphics there have been several attempts to simulate hydraulic erosion processes for the purpose of producing more realistic-looking terrains and create animations of surface deformation by fluids. These erosion simulations, while they do generate realistic terrains, do not model erosion, sediment transport, and deposition with real physical accuracy, and very few authors have attempted to validate their results in any way.

\subsection{Erosion Models}

Several erosion models have been presented, ranging from entire ecosystem models to small-scale watersoil boundary models.

\subsubsection{Ecosystem Models}

Two large scale ecosystem models use erosion modelling to simulate the behavior of an ecosystem. The The Integrated Biosphere Simulator (IBIS) model contains four modules modelling vegetation and land surface, arranged in a hierarchical framework (Foley et al. 1996). Each module runs on a separate time step, ranging from minutes to years. The model incorporates six soil layers, adjusting each layer's moisture content, ice level, and soil temperature, and the layers allow plants to draw water out. The Precision Agriculture-Landscape Modelling System (PALMS) divides landscapes in to two different systems, a gridbased terrain model and a layered soil system that uses the IBIS soil model described above (Moiling et al. 2005).

\subsubsection{Soil Loss}

Hanson et al. (2005) conducted seven overtopping erosion tests on large-scale physical cohesive embankment models, testing three soil types, two sand and one clay. From the tests, they determined a fourstage erosion process that occurs during overtopping of an embankment. Wang and Kahawita (2003) presented a two-dimensional mathematical model of ero- sion of the profile of an earthen embankment during overtopping. Similarly, Wang et al. (2006) presented a two dimensional mathematical model for the erosion of an embankment and compare it to test cases.

\subsubsection{Erodibility}

The "erodibility" of soil is generally defined as the ratio of the rate the soil erodes to the velocity of the water causing the erosion. Erodibility is an attempt to define a single parameter that will describe the behavior of a soil type under erosion conditions. Along similar lines, Hanson (1991) attempted to tie many standard erosion formulas together with the development of the "Jet Index", in which a jet of water is buried in soil and the rate of the soil's erosion is measured. Annandale (1995) linked several soil parameters and how they affect the energy gained or lost by the soil when a jet of water is run over it together through a series of tests, defining a new parameter, the "erodibility index".

Brandimarte et al. (2006) presented a Monte Carlo simulation method for determining the likelihood of reaching the critical scour depth to find the probability of reaching scour depth. Wan and Fell (2004) described the development of two new erosion rate tests, known as the Hole Erosion Test (HET) and the Soil Erosion Test (SET), which measure a soil's erodibility.

Each of the above methods for determining a soil's erodibility lack practicality for use small-scale erosion simulations. Briaud et al. (2008) strayed from a traditional definition of a soil's erodibility because water velocity varies throughout the flow field and the velocity at the soil-water interface is technically 0 . Instead, erodibility is treated as a function that is based on the hydraulic shear stress, or pull of the water on the soil, changing with water velocity so that it can be defined along the water/soil boundary, which incorporates the geometry of the soil as well as the water's properties along the flow field. Briaud's erosion model is ideal for small-scale erosion simulation because it allows for a parameter to be applied to the soil as a field over the geometry.

\subsection{Erosion Simulations in Computer Graphics}

Many erosion simulations have been performed in computer graphics, mainly focusing on generating realistic or interesting looking terrains.

\subsubsection{Terrain Development}

Musgrave et al. (1989) performed a basic erosion simulation on a height field in which each grid space stored an altitude, a height of water, and a layer of sediment, and sediment transport and deposition is performed on a cell-by-cell basis. Kelley et al. (1988), devised a way to produce fractal terrain from forming a stream network and growing the terrain up around 


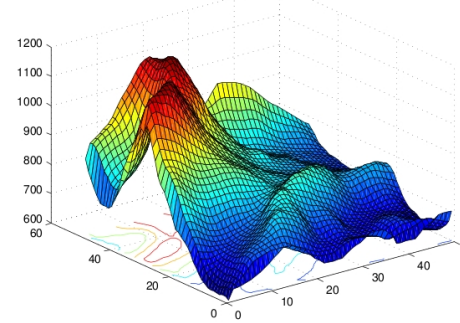

a)

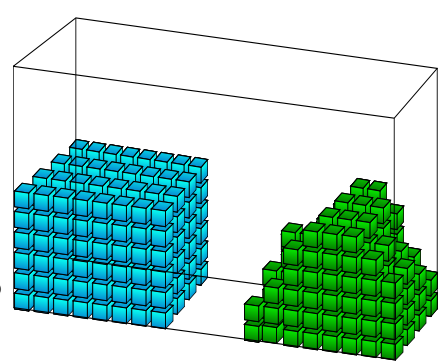

b)

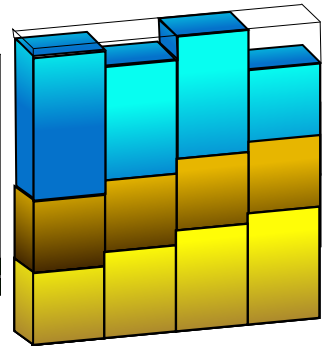

c)

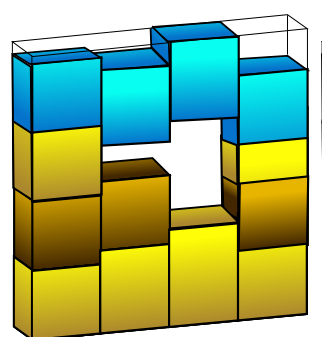

d)

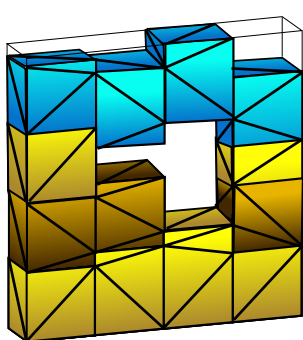

e)

Figure 3: Surface and Volumetric Data Structures: a) single-valued height field, b) voxel grid, c) layered height field d) our new Segmented Height Field (SHF), e) interpolated tetrahedrization of SHF.

it. Later attempts at terrain generation, such as Chiba et al. (1998), incorporate erosion simulation involving fluid simulation to carry sediment downstream using a fluid velocity field on a height field.

\subsection{Physically-Based Erosion Simulation}

Beyond terrain generation, research in computer graphics has also focused on erosion for the purpose of animation. Efficient algorithms that can be updated and changed dynamically are essential for erosion simulations. Three data structures dominate erosion research: height field (or height map), a layered height field, or a voxel grid (Fig. 3). Height fields are simply a grid array of heights, where each grid space allows for a single height value, and the terrain is generated by connecting these height values through some type of interpolation scheme. Height fields, like the one used by Benes and Arriaga (2005), are easily compressible, and allow for fairly simple surface extraction. However, they do not allow for layered terrains.

A voxel based terrain representation allows for immediate coupling with many fluid simulation techniques (such as Navier-Stokes), as these are often based on a voxel grid. Voxel-based terrain representations allow for multiple soil layers, different soil parameters, caves and undercuts, and volume information. Tetrahedral meshes are another volumetric representation that builds the model out of tetrahedra. This representation is more accurate in representing the model's surface than a voxel grid, which is limited to the grid resolution in three dimensions.

Benes and Forsbach (2001) presented a layered height field that combined several advantages of each of height fields and voxel grids. The terrain is divided into a two dimensional grid, like a height field, but each grid space contains an array of heights. This representation allows for several different soil types, a surface can be easily extracted for visualization and simulation purposes, the precision is arbitrary, and caves and undercuts are possible. The layered height field, however, does not allow for dynamic ordering of the layers, so new layers cannot be formed and if a particular soil type does not appear in a grid column then its value has zero depth, wasting space.

\subsubsection{Erosion Simulation}

Neidhold et al. (2005) presented an erosion simulation that coupled a terrain with a particle fluid simulator. The terrain was represented by a variation on a layered height field, where each grid space contains five layers representing different data layers: 3D acceleration, 3D velocity, fluid level, dissolved material layer, and soil height.Benes et al. (2006) presented a method for simulating hydraulic erosion on a homogeneous terrain where the terrain is modelled as a voxel grid where each cell in the grid has a state associated with it. The cell is either water, air, or soil and can transition from soil to water (with sediment) and back. Benes (2007) designed a shallow-water erosion simulation, and instead of continuing to use his layered height field he chose a simple two layer field, where there is a layer of water above a homogeneous layer of soil and a layer of regolith is allowed to develop between the two. Kristof et al. (2009) presented an erosion simulation using smoothed particle hydrodynamics (SPH). The soil, water, and soilwater boundary are all represented by particles, the soil and water particles have mass and velocity while the boundary particles are designed solely for the two phases to interact.

\subsection{Validation}

One of the major goals of our project is to present an erosion simulation that has been validated by physical experimentation. Many of the erosion models discussed above have provided case study validation. For the IBIS model, comparison testing was performed on the five test sites mentioned above, during which statistical analysis was performed, including root mean square error (RMSE) and mean biases for parameters such as soil moisture content, snow depth, and total monthly runoff. Wang and Kahawita (2003) compared their results to two test cases, one fictitious and one based on an actual flooding event.

All soil erodibility studies were based on both field and laboratory tests, and thus are validated by the methods used to procure them. Briaud used his values for soil erodibility for comparison to four case studies, calculating each case's failure probability, and in the case of New Orleans's levees during Hurricane Ka- 

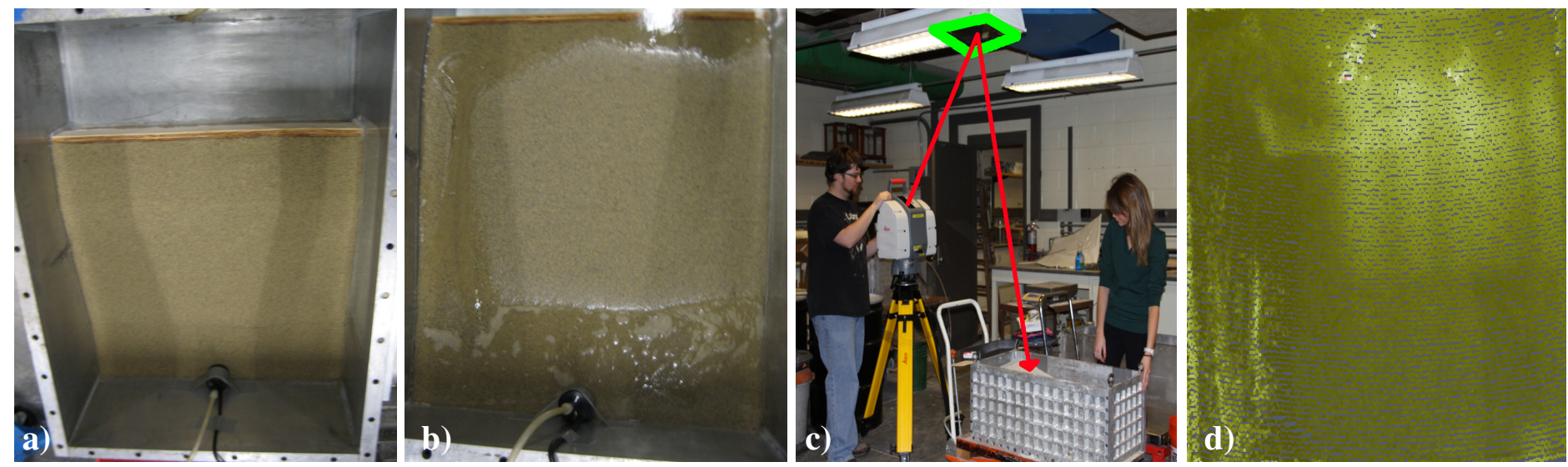

Figure 4: The formation of rills: a) initial experimental setup \& b) deeper channels within wet sand. c) The results are captured with a LIDAR scanner. d) A visualization of the 3D point cloud data.

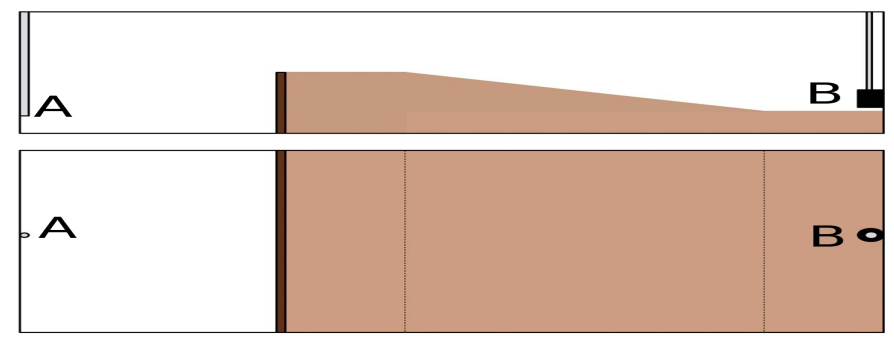

Figure 5: A schematic view of the experimental setup, including a profile view (top) and a top-down view (bottom). Water source is marked " $\mathrm{A}$ " and sink " $\mathrm{B}$ ".

trina, Briaud (2008) was able to use his erodibility model to categorize the soil from many of the levees that failed as "highly erodible".

To our knowledge, validation of computer simulations has not yet been accomplished, though it has been attempted with some success by the SODA project. Valette et al. (2006) presented work in which they take a sample of soil and model it as an extended cellular automaton on a grid, where each cell of the grid has many possible states, ranging from surface to sediment. Rain is randomly applied to the surface, and each surface cell's states is recalculated, allowing some cells to be detached from the surface and travel with the water. Deposition and sedimentation are handled via cell state changes.

What is most important about this work is that the authors performed an experiment with the same setup and visually compared the results of their simulation to their experimental results. Very little of the soil was actually transported and the rain dropped on the soil did not noticeably deform the soil surface. The authors' statistical analysis consists of determining where the terrains' heights differ, and no quantitative analysis was performed. We wish to extend on this validation to include statistical analysis and a firmer understanding about what it means for an erosion simulation to be physically accurate.

\section{PROCEDURES}

We have conducted preparatory 1 -g erosion tests in a laboratory.

\subsection{Soil Erosion Test}

Initial overtopping tests have been run on a half-levee. The embankment was anchored at one end by a $\frac{1}{2}$ inch thick piece of plywood sealed to the bottom of the tank using sillicone. The plywood was 6" high and 24 " wide. The box was 14 " x 24 " x 36 ", and the plywood separated the water source from the embankment crest. The plywood formed the source resevoir, which created rising water that overtopped the embankment crest, and its dimensions were 6" x 24 " $\mathrm{x}$ 8.5".

The embankment was made of well-graded medium sand, created from lifts (Fig. 4a). A lift is a thin layer of sand piled onto a compacted layer. To form the embankment, we compacted a 3" high layer of sand using a 4" by 4" wooden hand tamp, spread a lift over it, and compacted again. The 5:1 embankment measured 5" at the crest and 15.3" from crest to toe. A 5" x 24" floodplain was left at the toe of the embankment to collect the overtopping water with a small aquarium pump, placed $\frac{1}{2}$ " above the floodplain. A schematic of the setup is shown in Figure 5.

Water was run over the embankment with a flow rate of $11.1 \frac{\mathrm{mL}}{\mathrm{sec}}$. At 16 seconds from the time the water level in the source resevoir reached the top of the embankment's slope, the initial rill was formed (Fig. $4 \mathrm{~b}$ ), which we defined as eroded soil being evident from the crest to the toe. The water was run for 3 more minutes, at which point it was stopped and a scan of the surface was taken (Sec. 3.2), and the water was run for 8 more minutes. The formation of rills and gullies was closely observed.

\subsection{Data Collection}

Layer surface data is collected in the form of a point cloud via a 3D laser range scanner (Fig. 4c). This 3D point data is then run through a data preparation script that, for each scan, registers the points. It then aligns them to a regular grid in the XY plane, retaining the height values of the points, The points in a grid space have their heights averaged to acquire a single value to use in the data structure. If there are multiple soil layers in a single model, this procedure is repeated 


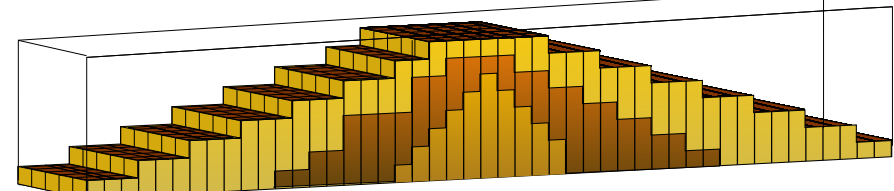

Figure 6: Our Segmented Height Field data structure is used to represent multi-layered levee.

for each one (as they are being assembled), generating a layered data structure (Sec. 3.3). In the future, change detection software will be run to determine where layers of different soils have formed, and anywhere where there is a layer of soil on top of another of the same soil type the layers are combined into one. The end result is a grid in which each cell contains an array of soil layers with heights and depths. This data is then loaded in to our data structure. Figure $4 d$ shows a top-down view of the data of a single layer of points captured by the LIDAR scanner, including their color information.

\subsection{Data Structure}

Our new data structure is the Segmented Height Field (SHF), shown in Figure 3d. It is similar to the layered height field, described earlier, in that it is a grid of cells, and each cell contains a list of the layers of soil found in that column. In our structure, each cell (referred to as a column) contains a list of soil segments (a rectilinear block of soil). Segments of the same soil type in adjacent grid columns that have overlapping intervals in height create a layer.

Several important differences exist between the layered height field and the SHF. First, SHF allows for layers to be dynamically added and removed throughout the simulation, making accurate re-deposition significantly easier. It is not possible to tell a priori where sediment will land and where new layers will have to be added on top of existing ones, so defining the soil layers before the simulation starts is impractical. Second, a layered height field does not allow for two layers of the same soil type to exist in the same list in one grid cell, whereas in our representation these layers are added automatically. Because there are no restrictions with regards to a soil segment's location within the column SHF naturally supports overhangs and air pockets, which can be modelled either implicitly by gaps in data or explicitly as air layers.

Furthermore, each SHF segment can have spatially varying soil parameters; for example, moisture content. Yet our SHF retains a key advantage of height fields: the layer height is not limited in resolution as it is for a voxel grid. Finally, the SHF can be more memory efficient than either a voxel grid or a layered height field. Voxel grids store redundant data when representing layers that are thick relative to the underlying grid. And, layered height fields are inefficient for accurate representation of complex soil re-deposition, because the addition of new layers is global rather than local.

\subsubsection{Interpolation and Visualization}

For visualization and simulation purposes, it is necessary to be able to generate an interpolated volume from our SHF. To do this, we generate a geometry in the form of a tetrahedral mesh from the data, allowing us to extract surface information, like slope, as well as generate surface normals for visualization (Fig. 3c). These not only improve the quality of the resulting visualization, but also will yield more accurate physical simulations by allowing water to flow smoothly down the embankment's inclined slopes and through channels cut within the soil.

\section{RESULTS}

The water overflowed from the reservoir and flowed through the soil ("groundwater" flow, seepage, etc.), thereby saturating the soil, and slowly flowed over the crest of the embankment on the surface. To a degree, surface tension was evident, as the water on the surface of the crest had boundaries (i.e. the water did not just come over the top in one big sheet of water). Once the water had crossed the crest on the surface, rill initiation along the left side of the embankment was seen, beginning at the heel of the slope (where edge of crest and edge of slope meet) and eroding its way to the toe of the slope. This formed the primary rill on the slope. Secondary, or tributary, rills formed and contributed to the main rill, but the water tended to continue to erode the first rill rather than form a new main rill. Once rilling began on the slope, the water began eroding a channel that receded across the crest from the heel of the slope towards the plywood, so the rill on the slope went one direction and the channel on the crest went in the opposite direction, but in line with the rill on the slope.

Typical results of our physical erosion modelling are shown in Figure 4a\&b. Rills form in the highly erodible sand of the test embankment, following the direction of the water flow. A LIDAR scan of these results are shown in Figure 4d. These geometrical features are accurately captured in the data.

\section{FUTURE WORK}

We will perform more detailed analysis of our results. We will also extend our embankment test models to include clayey soils, sand-clay mixtures, silts, layered materials, and other fine-grained soils. Our infrastructure allows layering and complex geometries with different crest widths and slope angles. Future erosion tests will be performed on a geotechnical centrifuge, allowing simulation and understanding of structures that scale to the size of actual embankments. We will use change detection software to gather and process data for multiple layers of soil, allowing us to simulate these more complex soil models in software. 
We are currently building a smoothed particle hydrodynamics simulation engine which will allow us to implement our erosion model. While studying the low-level erosion processes on physical soil models, we will also use our data capturing capabilities to build the same soil structures in software and run erosion simulations on them. Then we will use statistical analyses to compare each physical test to a collection of digital simulations. The results of these analyses will allow us to determine to what extent the erosion model can be validated with physical tests and will also suggest how to extend the erosion model for more complex scenarios.

\section{ACKNOWLEDGEMENTS}

This research was partially supported by NSF grant CMMI-0835762.

\section{REFERENCES}

Annandale, G. W. (1995). Erodibility. Journal of Hydraulic Research 33(4), 471-494.

Benes, B. (2007). Hydraulic erosion by shallow water simulation. The 4th Workshop on Virtual Reality Interaction and Physical Simulation - VRIPHYS'07.

Benes, B. and X. Arriaga (2005). Table mountains by virtual erosion. Eurographics Workshop on Natural Phenomena 2005, 33-40.

Benes, B. and R. Forsbach (2001). Layered data representation for visual simulation of terrain erosion. In Proceedings of the 17th Spring conference on Computer graphics.

Benes, B., V. Tesinsky, J. Hornys, and S. K. Bhatia (2006). Hydraulic erosion. Computer Animation and Virtual Worlds 17(2), 99-108.

Brandimarte, L., A. Montanari, J.-L. Briaud, and P. D'Odorico (2006). Stochastic flow analysis for predicting river scour on cohesive soils. Journal of Hydraulic Engineering 132(5), 493-500.

Briaud, J. L. (2008). Case histories in soil and rock erosion: Woodrow Wilson Bridge, Brazos River meander, Normandy Cliffs, and New Orleans levees. Journal of Geotechnical and Geoenvironmental Engineering 134(10), 1425-1447.

Briaud, J. L., H.-C. Chen, A. V. Govindasamy, and R. Storesund (2008). Levee erosion by overtopping in New Orleans during the Katrina Hurricane. Journal of Geotechnical and Geoenvironmental Engineering 134(618).

Chiba, N., K. Muraoka, and K. Fujita (1998). An erosion model based on velocity fields for the visual simulation of mountain scenery. The Journal of Visualization and Computer Animation 9(4), 185194.

Foley, J. A., I. C. Prentice, N. Ramankutty, S. Levis, D. Pollard, S. Sitch, and A. Haxeltine (1996). An integrated biosphere model of land surface processes, terrestrial carbon balance, and vegetation dynamics. Global Biogeochemistry Cycles 10, 603-628.
Hanson, G. J. (1991). Development of a jet index to characterize erosion resistance of soils in earthen spillways. Transactions of the ASAE 34(5), 20152020.

Hanson, G. J., K. R. Cook, and S. L. Hunt (2005). Physical modeling of overtopping erosion and breach formation of cohesive embankments. Transactions of the ASAE 48(5), 1783-1794.

Kelley, A. D., M. C. Malin, and G. M. Nielson (1988). Terrain simulation using a model of stream erosion. In Proceedings of the 15th Annual Conference on Computer Graphics and interactive Techniques, pp. 263-268.

Kristof, P., B. Benes, J. Krivanek, and O. St'ava (2009). Hydraulic erosion using smoothed particle hydrodynamics. Computer Graphics Forum 28(2), 219228.

Moiling, C. C., J. C. Strikwerda, J. M. Norman, C. A. Rodgers, R. Wayne, C. L. Morgan, G. R. Diak, and J. R. Mecikalski (2005). Distributed runoff formulation designed for a precision agricultural-landscape modeling system. Journal of the American Water Resources Association 41(6), 1289-1313.

Musgrave, F. K., C. E. Kolb, and R. S. Mace (1989). The synthesis and rendering of eroded fractal terrains. In Proceedings of the 16th annual conference on Computer graphics and interactive techniques, pp. 41-50.

Neidhold, B., M. Wacker, and O. Deussen (2005). Interactive physically based fluid and erosion simulation. In Eurographics Workshop on Natural Phenomena, pp. 25-32.

Seed, R., P. Nicholson, R. Dalrymple, J. Battjes, R. Bea, G. Boutwell, J. Bray, B. D. Collins, L. Harder, J. Headland, M. Inamine, R. Kayen, R. Kuhr, J. M. Pestana, R. Sanders, F. SilvaTulla, R. Storesund, S. Tanaka, J. Wartman, T. F. Wolff, L. Wooten, and T. Zimmie (2005). Preliminary report on the performance of the New Orleans levee systems in Hurricane Katrina. http: //www.berkeley.edu/news/media/releases/ 2005/11/leveereport_prelim.pdf.

Valette, G., S. Prevost, L. Lucas, and J. Leonard (2006). SoDA project: A simulation of soil surface degradation by rainfall. Computers \& Graphics 30(4), 494506.

Wan, C. F. and R. Fell (2004). Investigation of rate of erosion of soils in embankment dams. Journal of Geotechnical and Geoenvironmental Engineering 130(4), 373-380.

Wang, P. and R. Kahawita (2003). Modeling the hydraulics and erosion process in breach formation due to overtopping. In Sedimentation and Sediment Transport, Proceedings, pp. 211-220.

Wang, P., R. Kahawita, A. Moktari, T. M. Phat, and T. T.Quach (2006). Modeling breach formation in embankments due to overtopping. Transactions of the International Congress on Large Dams 22(3), 377-396. 